\title{
FAILURE OF POTASSIUM CANRENOATE TO ALTER EXPERIMENTALLY INDUCED DIGITALIS ARRHYTHMIAS *
}

\author{
Benedict R. LUCCHESI and Nancy R. HALEY ** \\ The University of Michigan Medical School, \\ Department of Pharmacology, Ann Arbor, Michigan 48104, U.S.A.
}

\begin{abstract}
B.R. LUCCHESI and N.R. HALEY, Failure of potassium canrenoate to alter experimentally induced digitalis arrhythmias, European J. Pharmacol. 22 (1973) 256-262.

The antiarrhythmic effects of potassium canrenoate were examined under in vivo and in vitro conditions. In the pentobarbital anesthetized dog, ventricular tachycardia was produced by the i.v. administration of ouabain, $64.4 \pm$ $3.7 \mu \mathrm{g} / \mathrm{kg}$. Potassium canrenoate was administered in increments of $10 \mathrm{mg} / \mathrm{kg}$ until a total dose of $30 \mathrm{mg} / \mathrm{kg} \mathrm{had}$ been achieved. 1 animal developed ventricular fibrillation and in the remaining 7 dogs, the ouabain-induced ectopic focus was not suppressed. However, in $3 \mathrm{dogs}$, a sinus or junctional tachycardia occurred after potassium canrenoate was administered and the rates exceeded that of the ventricular focus. Stimulation of the distal right vagus, however, resulted in a suppression of the supraventricular rhythm and a reappearance of the ventricular ectopic focus. In the rabbit perfused isolated heart, potassium canrenoate $\left(10^{-3} \mathrm{M}\right)$ failed to prevent or reverse acetylstrophanthidininduced arrhythmias. It is concluded that potassium canrenoate fails to suppress or reverse the cardiotoxic effects of digitalis in the intact dog heart or in isolated heart muscle preparations. In addition, sodium canrenoate does not prevent the positive inotropic action of oubain.
\end{abstract}

$\begin{array}{lll}\text { Sodium canrenoate } & \text { Acetylstrophanthidin } & \text { Ventricular tachycardia } \\ \text { Ouabain } & \text { Papillary muscles } & \text { Junctional tachycardia }\end{array}$

\section{Introduction}

Potassium canrenoate [soldactone, or potassium 3-(3-oxo-17ß-hydroxy-4,6-androstadien-17 $\alpha$-yl) propanoate], a diuretic which specifically antagonizes aldosterone, has been reported to be effective in preventing and in terminating experimentally induced digitalis arrhythmias (Yeh and Lazzara, 1971; Yeh et al., 1971; Yeh and Sung, 1972; Yeh et al., 1972). Yeh and Lazzara (1971) reported that potassium canrenoate in a concentration of $5 \times 10^{-5}$ restored the resting membrane potential, the action potential overshoot, $\mathrm{dV} / \mathrm{dt}$, and excitability in canine Purkinje fibers intoxicated with ouabain. Potassium canrenoate $(0.5 \mathrm{mEq}$, i.v.) was reported to successfully abol-

\footnotetext{
* This study was supported by U.S. Public Health Service Research Grant HE-05806-12.

** Nancy R. Haley is an Undergraduate Cardiovascular Research Trainee on the Cardiovascular Research Training Grant, National Institutes of Health Training Grant HE-05682.
}

ish ouabain-induced ventricular tachycardia in 6 of 11 dogs (Yeh and Sung, 1972). All potassium canrenoate conversions took place within one minute after the intravenous administration of the drug and were noted to be an 'all or none phenomenon' and the conversion was said to be 'permanent'. The observations in the intact dog and upon isolated Purkinje fiber preparations were interpreted as suggesting that potassium canrenoate was a specific antagonist to the electrophysiological effects of digitalis on the cardiac cell membrane (Yeh and Lazzara, 1971); a conclusion that was supported by the inability of potassium canrenoate to reverse arrhythmias secondary to acute coronary artery ligation or to catecholamines (Yeh et al., 1972 b).

The present study was undertaken in an effort to further explore the antiarrhythmic properties of potassium canrenoate. The results of this investigation have led to the conclusion that potassium canrenoate 
does not possess a specific antiarrhythmic effect against digitalis-induced cardiac rhythm disturbances and that previously reported success with potassium canrenoate might have been misinterpreted due to the ability of the drug to enhance sinus and/or junctional pacemaker activity in the digitalis intoxicated heart.

\section{Materials and methods}

\subsection{Ouabain-induced arrhythmias in the anesthetized $\operatorname{dog}$}

Male mongrel dogs $(8.0-12.0 \mathrm{~kg})$ were anesthetized with pentobarbital sodium, $30 \mathrm{mg} / \mathrm{kg}$, i.v. Blood pressure was measured from the left femoral artery with a Statham transducer. All drugs were administered into the cannulated left external jugular vein. The right vagus nerve was sectioned and its distal end was stimulated with $1.0 \mathrm{msec}$ square-wave stimuli at a frequency of $20-40 \mathrm{cps}$ at $8.0-10.0 \mathrm{~V}$.

Ventricular tachycardia was induced by the administration of ouabain, $40 \mu \mathrm{g} / \mathrm{kg}$ i.v., followed in $30 \mathrm{~min}$ by $20 \mu \mathrm{g} / \mathrm{kg}$ and every $15 \mathrm{~min}$ thereafter by an additional $10 \mu \mathrm{g} / \mathrm{kg}$ until ventricular tachycardia developed (Lucchesi and Hardman, 1961).

The criteria used to determine antiarrhythmic activity were: (1) reversion to normal sinus rhythm for a period of not less than $30 \mathrm{~min}$ and (2) the failure of right vagal stimulation to expose automatic ectopic ventricular activity during the period of vagal-induced sino-atrial arrest.

Lead II electrocardiograms were monitored continuously on an oscilloscope and all recordings were made on a Grass model 7 polygraph.

\subsection{Acetylstrophanthidin-induced arrhythmias in the rabbit isolated heart}

The rabbit isolated heart was perfused according to the Langendorff method. Male rabbits (New Zealand strain), $1.8-2.0 \mathrm{~kg}$ in weight were killed by a cervical dislocation and their hearts were removed quickly. The perfusion solution was of the following composition, expressed as mmoles/1: $\mathrm{NaCl} 171.1, \mathrm{KCl}$ 2.8, $\mathrm{CaCl}_{2} \cdot 2 \mathrm{H}_{2} \mathrm{O} 2.0, \mathrm{MgCl}_{2} \cdot 6 \mathrm{H}_{2} \mathrm{O} 2.1$, dextrose 10.0, and THAM buffer, 4.1. The final solution was adjusted to a $\mathrm{pH}$ of 7.4 by the addition of $1 \mathrm{~N} \mathrm{HCl}$.
The heart was enclosed in a double-walled glass chamber; the heart and perfusion medium were maintained at a temperature of $30^{\circ} \mathrm{C}$. The perfusion solution was recycled and oxygenated by passing $100 \%$ oxygen into the reservoir containing the perfusion solution.

Electrocardiographic recordings were made on a Beck-Lee electrocardiograph and were monitored continuously on an oscilloscope.

Arrhythmias were produced in the isolated heart through the use of acetylstrophanthidin added to the reservoir in a concentration of $640 \mu \mathrm{g} / 1$ which resulted in a rhythm disturbance within $2-5 \mathrm{~min}$. The arrhythmia was characterized as a nodal tachycardia or nodal (junctional) rhythm with retrograde P-wave conduction. Longer periods of exposure to acetylstrophanthidin resulted in multifocal ventricular tachycardia and ventricular fibrillation.

The time required for the appearance of nodal tachycardia or nodal rhythm (latent period) after the administration of acetylstrophanthidin was determined three consecutive times. Between each trial, the heart was washed with drug-free perfusion solution for a period of $20 \mathrm{~min}$. The fourth exposure to acetylstrophanthidin and the time to arrhythmia was done in the presence of potassium canrenoate added to the perfusion medium prior to the addition of acetylstrophanthidin and the latent period determined as before.

In addition to demonstrating the ability of a compound to prevent the onset of the acetylstrophanthidin arrhythmia, it was also possible to demonstrate the ability of a compound to suppress an already established arrhythmia. Once the acetylstrophanthidininduced arrhythmia had been established, potassium canrenoate was added to the perfusion solution over a period of time to a maximum concentration of $10^{-3} \mathrm{M}$.

\subsection{Cat isolated papillary muscle preparations}

Cats of either sex, $1.5-2.0 \mathrm{~kg}$ were anesthetized with pentobarbital sodium given intraperitoneally in a dose of $30 \mathrm{mg} / \mathrm{kg}$. The hearts were rapidly excised and placed in oxygenated, modified Krebs solution warmed to $37^{\circ} \mathrm{C}$. Right ventricular papillary muscles were dissected free and mounted in muscle chambers containing $50 \mathrm{ml}$ of modified Kreb's solution maintained at $37^{\circ} \mathrm{C}$ and oxygenated with a mixture of 
$95 \% \mathrm{O}_{2}-5 \% \mathrm{CO}_{2}$; the $\mathrm{pH}$ being 7.4. The non-tendinous ends of the muscles were attached in a Lucite clip and the upper end of the muscles were attached by braided non-capillary silk to a FT-03 force displacement transducer. The muscles were stretched by a resting force which was at the peak of their active length-tension curves and were maintained at this length throughout the study. The isometric muscles were stimulated via field electrodes with square-wave pulses of $0.5-\mathrm{msec}$ duration at a rate of $12 \mathrm{~min}^{-1}$ with a current $10 \%$ above threshold delivered from a $104 \mathrm{~A}$ American Electronics Laboratory Stimulator. All recordings were made on a Grass Model 7 Polygraph.

The composition of the bath medium was as follows, expressed as $\mathrm{mM}: \mathrm{Na}^{+} 143, \mathrm{~K}^{+} 5.9, \mathrm{Ca}^{2+} 2.5$, $\mathrm{Mg}^{2+} 1.9, \mathrm{Cl}^{-} 126, \mathrm{H}_{2} \mathrm{PO}_{4}^{-} 1.19$, dextrose 5 .

At the end of each experiment, the length of each muscle was determined, the muscles were weighed and their cross sectional area was calculated according to the formula:

$$
\begin{aligned}
& \text { cross sectional area }\left(\mathrm{mm}^{2}\right)=\text { mass }(\mathrm{mg}) / \\
& \operatorname{spG}\left(1.051 \mathrm{mg} / \mathrm{mm}^{3}\right) \times \text { length }(\mathrm{mm})
\end{aligned}
$$

Data were expressed as force in $\mathrm{g} / \mathrm{mm}^{2}$ cross sectional area. Maximal developed force was determined by electrical paired pacing. The data were then expressed as a percentage of the maximal developed force.

Statistical analysis of all data was done by using Student's $t$-test for paired comparisons (Snedecor, 1956).

The potassium and sodium salts of canrenoate used in this study were obtained from Dr. W. Scott Smith, Searle Laboratories, Chicago, Illinois.

\section{Results}

\subsection{Effects of potassium canrenoate on ouabain- induced ventricular tachycardia}

Ouabain was administered to each of $8 \mathrm{dogs}$ in an average dose of $64.4 \pm 3.7 \mu \mathrm{g} / \mathrm{kg}$, i.v., which resulted in a sustained ventricular tachycardia in each of the animals. The abnormal rhythm was allowed to continue uninterrupted for at least $20 \mathrm{~min}$ to insure against spontaneous reversal of the rapid ventricular ectopic rhythm. Stimulation of the distal end of the cut right vagus nerve failed to alter the ouabain- induced ectopic focus. Administration of potassium canrenoate, given i.v. as 3 injections of $10 \mathrm{mg} / \mathrm{kg}$ over a period of $20 \mathrm{~min}$ (total dose equal to 30 $\mathrm{mg} / \mathrm{kg}$ ) failed to restore normal sinus rhythm in any of the animals. One of the 8 dogs which had received $60 \mu \mathrm{g} / \mathrm{kg}$ of ouabain and had developed a multifocal ventricular tachycardia ultimately developed ventricular fibrillation after receiving potassium canrenoate. Although normal sinus rhythm was not reestablished in any of the animals, there was the occurrence of a sino-atrial and/or junctional tachycardia in 3 experiments one of which is shown in fig. 1 . In each case, the rate of the sino-atrial or junctional tachycardia exceeded the rate of discharge of the ventricular ectopic rhythm. Upon stimulation of the distal end of the right vagus nerve, however, the rate of the supraventricular rhythm slowed sufficiently so as to permit the ventricular focus to become the dominant rhythm. Stimulation of the vagus nerve in the remaining experiments was without effect on the ventricular ectopic focus which continued unaltered in spite of the administration of potassium canrenoate. The subsequent administration of propranolol (2.3 \pm $0.1 \mathrm{mg} / \mathrm{kg}$ ) to the 7 remaining animals restored normal sinus rhythm in every instance. Furthermore, stimulation of the distal end of the right vagus nerve suppressed sino-atrial nodal pacemaker activity without unmasking ventricular pacemakers since propranolol has the ability, unlike potassium canrenoate, to suppress digitalis-induced automaticity. The administration of insulin ( 80 units, i.v.) restored ventricular tachycardia in each instance and served to demonstrate that ouabain was still present in a concentration sufficient to induce disturbances in ventricular rhythm. The mechanism by which insulin causes a return of the ectopic focus involves the ability of the hormone to enhance cellular uptake of glucose which results in a decrease in plasma potassium. The resulting hypokalemia intensifies the toxic effect of the digitalis glycoside.

\subsection{Effects of potassium canrenoate on acetylstro- phanthidin-induced arrhythmias}

5 rabbit isolated hearts were perfused by the Langendorff method. Each heart was exposed to acetylstrophanthidin, $640 \mu \mathrm{g} / \mathrm{l}, 3$ consecutive times for a total of 15 control determinations. The average 

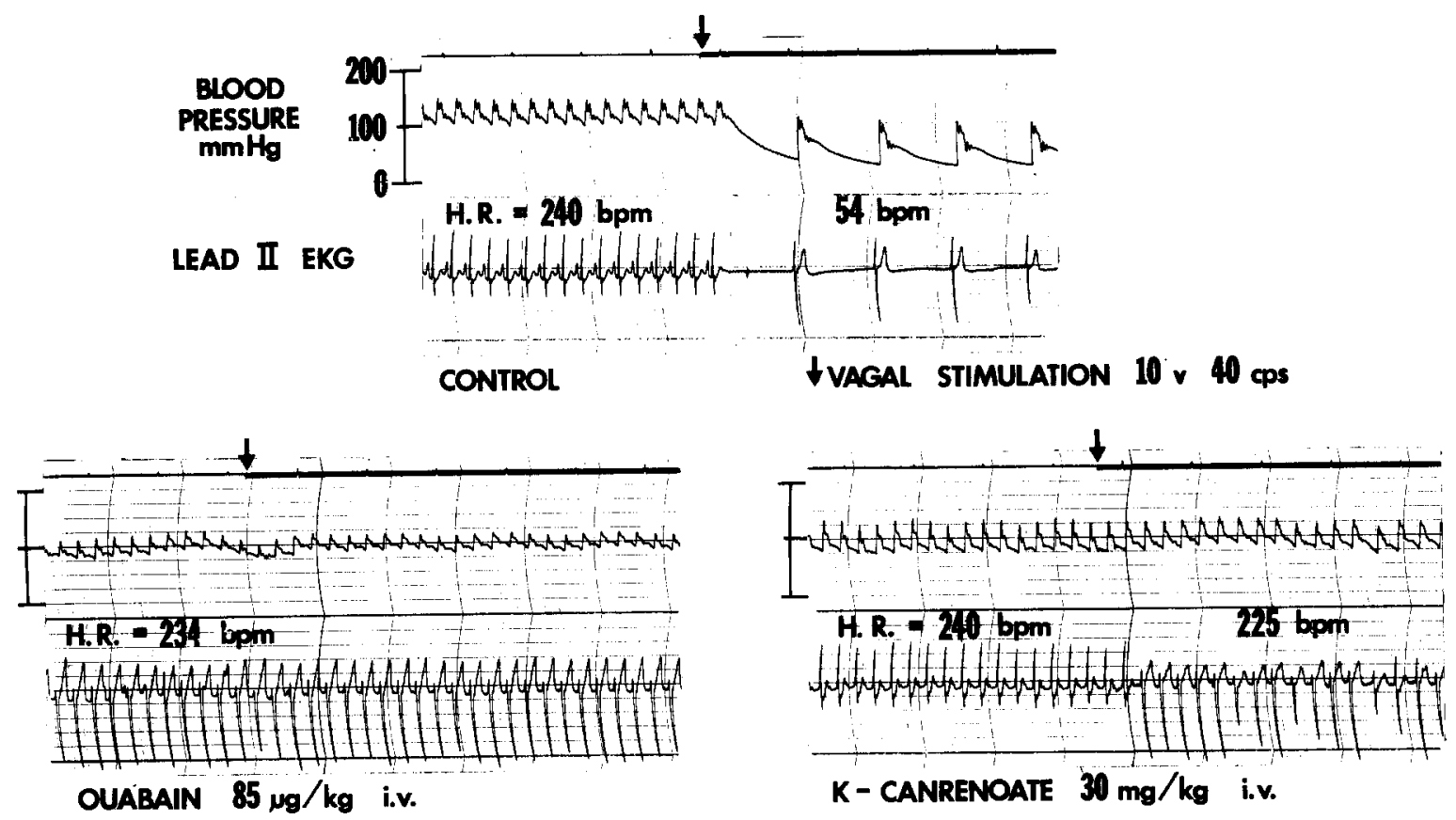

K - CANRENOATE $30 \mathrm{mg} / \mathrm{kg}$ i.v.

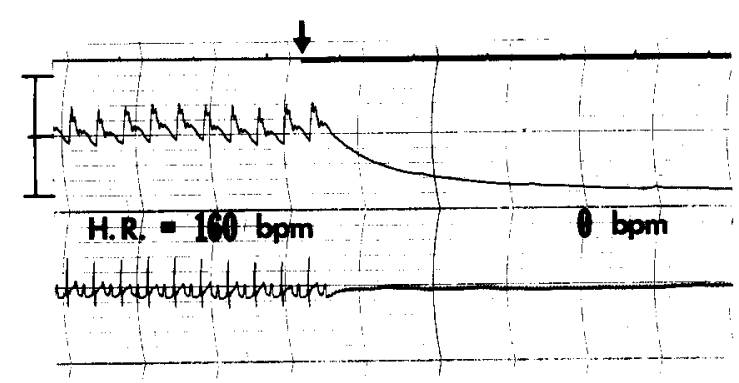

PROPRANOLOL $2 \mathrm{mg} / \mathrm{kg}$ i.v.

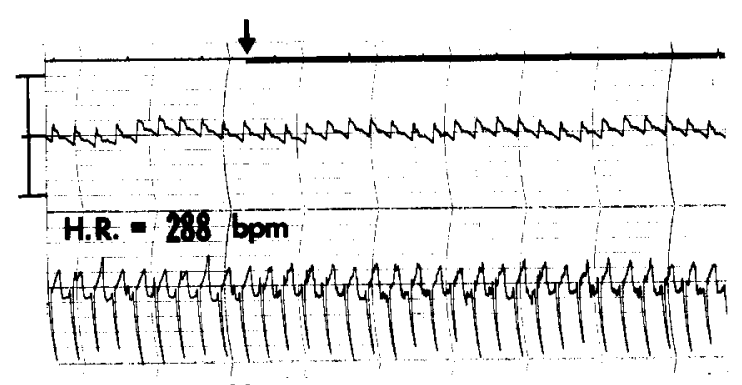

INSULIN 80 units i. $\mathbf{~}$

Fig. 1. Failure of potassium canrenoate to suppress the ouabain-induced ventricular ectopic focus in the canine heart. Each panel shows the arterial blood pressure and the Lead II electrocardiogram. The upper central panel is a control tracing illustrating the ability of right vagal stimulation to suppress the supraventricular pacemaker. The center left hand panel shows the effects of a toxic dose of ouabain. The ouabain-induced ventricular tachycardia is not influenced by stimulation of the right vagus. The ventricular rate is 234 beats $/ \mathrm{min}$. After the administration of potassium canrenoate $(30 \mathrm{mg} / \mathrm{kg}$, i.v. $)$, there is an abrupt change in the electrocardiogram from that of a ventricular tachycardia to that of a sino-atrial tachycardia with a rate of $240 \mathrm{beats} / \mathrm{min}$. Stimulation of the right vagus nerve, however, unmasks the ouabain-induced ventricular ectopic focus (rate 225 beats/min). The lower left hand panel shows the effects of propranolol in terminating the digitalis-induced arrhy thmia and a complete suppression of the ectopic focus as evidenced by the suppression of all pacemaker activity during vagal stimulation. The lower right hand panel shows a return of the ventricular ectopic pacemaker after the administration of insulin.

'latent period' before the onset of the arrhythmia was $185.6 \pm 18.8$ (S.E.M.) sec. When the hearts were simultaneously perfused with a medium containing potassium canrenoate, $10^{-3} \mathrm{M}$ and acetylstrophanthidin, $640 \mu \mathrm{g} / \mathrm{l}$, the time to arrhy thmia averaged 191.8 $\pm 37.5 \mathrm{sec}$ and was not significantly different from control $(p>0.5)$. Thus, potassium canrenoate did not prevent the development of acetylstrophanthidininduced toxicity in the rabbit isolated heart.

In a separate series of 5 rabbit isolated hearts, arrhythmias were produced by exposing the preparations to acetylstrophanthidin, $640 \mu \mathrm{g} / \mathrm{l}$. When the arrhy thmia had been established, potassium canrenoate was added to the perfusion solution in concentrations 

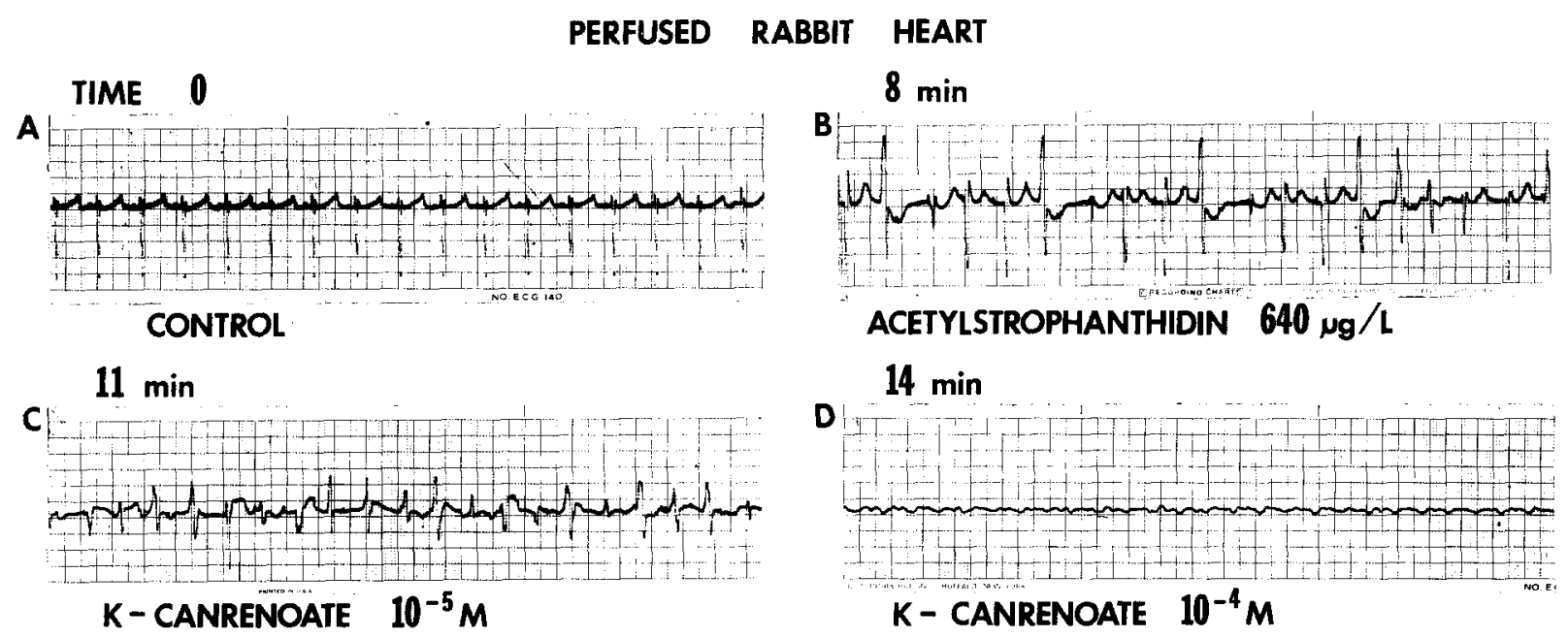

Fig. 2. The inability of potassium canrenoate to reverse the acetylstrophanthidin-induced arrhythmia in the rabbit isolated heart. Panel $\mathrm{A}$ is a control electrocardiogram. Panel B shows a multifocal ventricular tachycardia 8 min after acetylstrophanthidin was added to the perfusion medium. Panel $\mathrm{C}$ was taken $3 \mathrm{~min}$ after the addition of potassium canrenoate $\left(10^{-5} \mathrm{M}\right)$ and Panel $\mathrm{D}$ was made $3 \mathrm{~min}$ after the concentration of potassium canrenoate was increased to $10^{-4} \mathrm{M}$. As illustrated in the final tracing, the multifocal ventricular arrhythmia finally terminated in ventricular fibrillation.

of $10^{-5} \mathrm{M}, 10^{-4} \mathrm{M}$ and $10^{-3} \mathrm{M}$ with each addition being made at 3-min intervals. In no instance was there a return to normal sinus rhythm. Fig. 2 illustrates the sequence of events from one experiment in which ventricular fibrillation developed 3 min after potassium canrenoate, $10^{-4} \mathrm{M}$ had been added to the perfusion medium. In the remaining 4 hearts, potassium canrenoate, $10^{-3} \mathrm{M}$, did not alter the acetylstrophanthidin-induced arrhythmia which progressed in each case to a multifocal ventricular tachycardia and ventricular fibrillation.

\subsection{Effect of sodium canrenoate on cat isolated pap- illary muscles}

The sodium salt of canrenoate was used in this part of the study to circumvent the effects of the potassium ion upon developed isometric force. A total of 6 cat isolated papillary muscles was exposed to sodium canrenoate in concentrations of $10^{-6}$ to $10^{-3} \mathrm{M}$. Control isometric force averaged $61.2 \% \pm 6.0$ (S.E.M.) of the developed maximal force in response to paired pacing. Significant depression in isometric force was apparent at a sodium canrenoate concentra- tion of $10^{-4} \mathrm{M}$. At this concentration, developed isometric tension averaged $49.7 \% \pm 5.5$ of maximal isometric force $(p<0.001)$ with a further depression in contractility occurring at $10^{-3} \mathrm{M}$ sodium canrenoate where isometric force averaged $21.5 \% \pm 2.8$ of the maximal isometric force $(p<0.001)$. The subsequent addition of ouabain $\left(1.02 \times 10^{-6} \mathrm{M}\right)$ in the presence of $10^{-3} \mathrm{M}$ sodium canrenoate resulted in a positive inotropic effect which averaged $60.7 \% \pm 7.4$ of the maximal developed isometric tension. Thus, sodium canrenoate produces a significant negative inotropic effect in cat isolated papillary muscles. Furthermore, the digitalis glycoside, ouabain, is capable of reversing the negative inotropic action of sodium canrenoate which would suggest that the latter does not antagonize or prevent the positive inotropic effects of digitalis. It was of further interest, that 5 of the 6 papillary muscles exposed to ouabain $\left(1.02 \times 10^{-6} \mathrm{M}\right)$ and sodium canrenoate $\left(10^{-3} \mathrm{M}\right)$ ultimately developed signs of toxicity as evidenced by the development of automaticity. Thus, as in the previous studies in the intact canine heart and in the rabbit isolated heart, digitalisinduced toxicity could not be prevented by the potassium or sodium salts of canrenoate. 


\section{Discussion}

The present investigation dealing with the ability of potassium canrenoate to antagonize digitalisinduced arrhythmias has led to a conclusion that is in disagreement with that of Yeh et al. (1971), Yeh et al. (1972) and Yeh and Sung (1972). The previous studies had suggested that potassium canrenoate was an antagonist to the cardiotoxic actions of ouabain and possessed an 'all or none' effect (Yeh and Sung, 1972) as evidenced by the fact that 6 of the 11 ouabain-intoxicated animals were successfully converted. Reference to fig. 3 in the publication by Yeh and Sung (1972) may provide an answer to the discrepancy between their results and those obtained in the present study. It will be noted that the rate of the ventricular ectopic focus decreased from 200 to 190 per min soon after the administration of potassium canrenoate. The conversion to normal sinus rhythm which followed was at a rate of 200 per min, a rate which now exceeded that of the ventricular ectopic focus and thus became the dominant rhythm. A similar phenomenon was seen in the present investigation and is illustrated in fig. 1. The development of a sinoatrial or junctional rhythm with a rate in excess of the ventricular ectopic focus tended to mask the underlying ouabain-induced automaticity. Stimulation of the vagus nerve, however, which is capable of decreasing the rate of $\mathrm{S}-\mathrm{A}$ nodal and junctional rhythms permits the ventricular ectopic focus to become the dominant pacemaker. The present studies demonstrate that potassium canrenoate does not have the ability to suppress digitalis-induced automaticity in the intact dog heart and the 'all or none' effect reported by Yeh and Sung (1972) might be related to a masking of the ventricular ectopic focus by a supraventricular pacemaker with a faster rate of discharge. The results obtained with propranolol in the present study illustrate the importance of slowing the supraventricular pacemaker in order to demonstrate that the antiarrhythmic agent is capable of suppressing the ouabain-induced ectopic focus and that the conversion is not due to overdrive suppression of the ventricular pacemaker.

Studies in the isolated heart are in agreement with those in the intact canine heart. The acetylstrophanthidin-induced arrhythmia in the rabbit Langendorff heart preparation has been used successfully in this laboratory to evaluate the antiarrhythmic activity of several agents (Lucchesi and Hardman, 1961; Lucchesi, 1965; Lucchesi and Iwami, 1968) and has provided good correlation with in vivo results. The same correlation between in vivo and in vitro results exists with respect to the observations made with potassium canrenoate. The drug failed to prevent or delay the appearance of the acetylstrophanthidin-induced arrhythmia in the rabbit isolated heart. Furthermore, once an arrhythmia had been established, potassium canrenoate was unable to abolish the cardiotoxic effects of acetylstrophanthidin. In addition, the sodium salt of canrenoate produced a marked negative inotropic effect in cat isolated papillary muscles. The depressant effect of sodium canrenoate was reversed by ouabain, further demonstrating that canrenoate lacks a specific antagonistic action to the effects of digitalis as suggested by Yeh and Lazzara (1971). Yet to be resolved is the fact that Yeh and Lazzara (1971) have noted that potassium canrenoate restored the resting. membrane potential, action potential overshoot, $\mathrm{dV} / \mathrm{dt}$, and excitability in digitalis-poisoned Purkinje fibers. Whether or not this effect is specific for digitalis-induced alterations in the membrane action potential remains to be determined. In addition the recently reported observation by Yeh et al. (1972), that potassium canrenoate improves atrioventricular transmission in the digitalis-intoxicated heart could also provide an explanation for the apparent ability of the drug to restore normal sinus rhtyhm in the digitalis-intoxicated heart.

The results of these studies lead to the conclusion that potassium canrenoate, a specific antagonist of aldosterone, lacks specific antiarrhythmic activity against digitalis-induced arrhythmias.

\section{References}

Lucchesi, B.R., 1965, The effects of pronethalol and its dextro-isomer upon experimental cardiac arrhythmias, J. Pharmacol. Exptl. Therap. 148, 94.

Lucchesi, B.R. and H.F. Hardman, 1961, The influence of dichloro-isoproterenol (DCI) and related compounds upon ouabain and acetylstrophanthidin-induced arrhythmias, J. Pharmacol. Exptl. Therap. 132, 372.

Lucchesi, B.R. and T. Iwami, 1968, The antiarrhythm ic properties of ICI 46037 , a quaternary analog of propranolol, $J$. Pharmacol. Exptl. Therap. 162, 49. 
Snedecor, C.W., 1956, Statistical Methods, 5th ed. (Iowa State College Press, Ames, Iowa).

Yeh, B.K. and R. Lazzara, 1971, Potassium canrenoate: Electrophysiological effects of normal and digitalis poisoned canine Purkinje fibers, Pharmacologist 13, 224.

Yeh, B.K. and P.K. Sung, 1972, Prevention and abolition of oubain-induced ventricular tachycardia by potassium canrenoate in dogs, European J. Pharmacol. 19, 151.

Yeh, B.K., A.K. Saha, P.K. Sung and P. Samet, 1971, Saldac- tone (SC-14,266): Suppression of digitalis induced arrhythmias, Federation Proc. 30, 228.

Yeh, B.K., N. deGuzman, T. Pinakatt and P. Tao, 1972, Specific antagonism of chronic digoxin intoxication in awake dogs, Circulation 46, Suppl. II 238.

Yeh, B.K., P.K. Sung and A.K. Saha, 1972, Use of potassium canrenoate to suppress ouabain-induced ventricular arrhy thmias in dogs, Circulation Res. 31, 915. 